\title{
Profesionalización docente en el nivel superior, bajo el enfoque de competencias
}

\section{Teacher professionalization at the higher level, under the competence approach}

\author{
CANO-CORONA, Ariana $\dagger^{*}$, CANO-CORONA, Melina, FLORES-FARIAS, Sadi, y CISNEROS- \\ FLORES, Federico Jorge
}

Universidad Politécnica de Tlaxcala

ID $1^{\mathrm{er}}$ Autor: Ariana, Cano-Corona / ORC ID: 0000-0003-3456-2303

ID $1^{\text {er }}$ Coautor: Melina, Cano-Corona / ORC ID: 0000-0003-4172-5705

ID $2^{\text {do }}$ Coautor: Sadi, Flores-Farias / ORC ID: 0000-0003-4801-9635

ID $3^{\text {er }}$ Coautor: Federico Jorge, Cisneros-Flores / ORC ID: 0000-0002-0839-0352

DOI: $10.35429 / J H S .2019 .10 .3 .6 .11$

Recibido 09 de Octubre, 2019, Aceptado, 30 de Noviembre, 2019

\begin{abstract}
Resumen
En este trabajo se aborda un estudio parcial que se está llevando a cabo sobre las estrategias didácticas que se utilizan en la práctica docente de dos instituciones de educación superior en el Estado de Tlaxcala, y que trabajan bajo el enfoque por competencias. De igual manera se realiza un análisis de las competencias básicas que deben poseer los docentes de dichas instituciones para trabajar cualquier asignatura de los mapas curriculares establecidos a nivel superior bajo este mismo enfoque. A través de este estudio y su posterior análisis se expone la realidad de las prácticas que llevamos a cabo los docentes bajo el enfoque por competencias, una vez que se han aprobado reformas educativas centradas en la profesionalización de los docentes, mostrando que existen grandes retos, algunos avances y extensas áreas de oportunidad en el corto plazo. Evidenciando, que si bien el enfoque educativo por competencias se trabaja actualmente en todos los niveles, aún existen muchos vacíos entre los eslabones que componen la cadena del sistema educativo hasta llegar e impactar en el nivel superior.
\end{abstract}

Profesionalización, Docente, Competencias

\begin{abstract}
This paper deals with a partial study that is being carried out on teaching strategies that are used in the teaching practice of some institutions of higher education, which work under the competence approach. Likewise, an analysis is made of the basic competences that teachers must possess to work on any subject of the curricular maps established at a higher level under this same approach. Through this study and analysis the reality of teaching practices is exposed once educational reforms focused on the professionalization of teachers have been approved, showing the challenges, advances and areas of opportunity in the short term. Evidencing that although the educational approach by competences is currently being worked on at all levels, from basic education to higher education, there are still many gaps between the links that make up the education system chain
\end{abstract}

Tecaher, Professionalization, Higher education

Citación: CANO-CORONA, Ariana, CANO-CORONA, Melina, FLORES-FARIAS, Sadi, y CISNEROS-FLORES, Federico Jorge. Profesionalización docente en el nivel superior, bajo en el foque de competencias. Revista de Educación Superior. 2019 3-10: 6-11

\footnotetext{
* Correspondencia del Autor (Correo electrónico: ariana.cano@uptlax.edu.mx)

$\dagger$ Investigador contribuyendo como primer autor.
} 


\section{Introducción}

En este trabajo se aborda un estudio realizado en dos instituciones de educación superior que muestran los avances de la profesionalización docente, bajo en enfoque de competencias.

La educación siempre ha sido uno de los aspectos prioritarios a considerar en las políticas públicas de cualquier gobierno, ya que está demostrado que es un motor de desarrollo social, pero sobre todo impacta directamente en la economía de las naciones.

En América latina, la educación ha tenido un progreso importante, en el caso del nivel superior, en las últimas décadas ha crecido el número de instituciones de educación superior, pero sobre todo existe un crecimiento de la tasa poblacional y también el interés de los gobiernos por mejorar las condiciones sociales se ha incrementado.

En el caso de México, si bien es cierto que las últimas reformas educativas marca como uno de sus objetivos principales la profesionalización docente en todos sus niveles, ¿realmente se tiene algún avance en el nivel superior, respecto a este rubro?

En el primer apartado se aborda el escueto marco normativo que se tiene sobre la profesionalización docente en el nivel superior y posteriormente se realiza un trabajo de campo para observar si efectivamente se ha tenido un impacto de profesionalización en la planta docente de las instituciones, a partir de las últimas reformas educativas en el país.

Finalmente se discute la efectividad de la mencionada profesionalización docente a nivel superior en las instituciones de estudio y que probablemente también se observe a nivel general.

\section{Marco normativo de la profesionalización docente en nivel superior en México}

Actualmente en México se están llevando a cabo grandes cambios económicos, políticos, y sociales, dentro de los cuales se incluye la Reforma Educativa que en teoría menciona la mejora de la calidad de todos los niveles educativos, entre muchas otras cosas.
Sin embargo por Ley, actualmente la educación obligatoria solamente incluye hasta el nivel medio superior (2013), por lo tanto no existe un marco normativo y jurídico específico para establecer indicadores y criterios objetivos de la calidad en el nivel superior, hablando específicamente de Universidades Públicas. Si bien es cierto que existen organismos certificadores de la calidad en la educación superior (Comités Interinstitucionales para la Educación Superior CIEES, Consejo de Acreditación de la Enseñanza de la Ingeniería CACEI), estos basan sus estándares en los criterios establecidos a nivel global (Organización de la Naciones Unidas ONU, Organización para la Cooperación y el Desarrollo Económico OCDE, etc.).

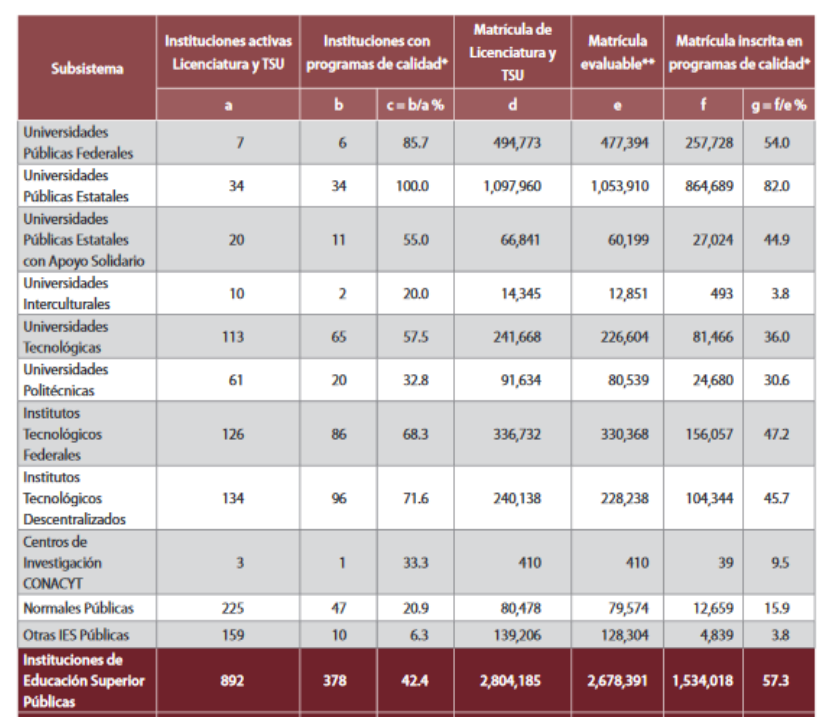

Tabla 1 Indicadores de matrícula inscrita en instituciones de educación superior acreditados por su calidad Fuente: ANUIES, con datos de los Formatos 911 de educación superior, CIEES y COPAES. Actualización: enero de 2018

Cabe destacar entonces que en el caso de las Universidades Públicas la mayoría de las investigaciones sobre educación se centran en los modelos educativos, así como en la calidad que se deben ofrecer, pero casi ninguno abarca el marco normativo que establezca los criterios de calidad efectiva y/o las competencias que deben cumplir los docentes de nivel superior.

Para el caso de las normales a partir del 2003 se desarrolla el Programa de Mejoramiento Institucional de las Escuelas Normales Públicas (PROMIN), (ver Tabla 1) posteriormente con el surgimiento de la Alianza por la Calidad de la Educación se da prioridad a la profesionalización de magisterio. 


\begin{tabular}{|l|c|}
\hline \multicolumn{1}{|c|}{ Nombre } & Año \\
\hline Ley federal de educación & 1973 \\
\hline $\begin{array}{l}\text { Acuerdo Nacional de Modernización } \\
\text { de la Educación Básica }\end{array}$ & 1992 \\
\hline Ley General de Educación & 1993 \\
\hline Carrera Magisterial Nacional para la & $1995-2008$ \\
\hline $\begin{array}{l}\text { Programa de los } \\
\text { Actualización Permanente } \\
\text { Maestros de Educación Básica en } \\
\text { Servicio }\end{array}$ & \\
\hline $\begin{array}{l}\text { Programa para la transformación y } \\
\text { fortalecimiento académico de las } \\
\text { Escuelas Normales }\end{array}$ & 1996 \\
\hline $\begin{array}{l}\text { Programa de Mejoramiento del } \\
\text { Profesorado }\end{array}$ & 1996 \\
\hline $\begin{array}{l}\text { Instituto Nacional para la Evaluación } \\
\text { de la Educación }\end{array}$ & 2002 \\
\hline $\begin{array}{l}\text { Programa de mejoramiento } \\
\text { institucional de las Escuelas Normales } \\
\text { Públicas }\end{array}$ & 2003 \\
\hline $\begin{array}{l}\text { Programa Estatal de Fortalecimiento } \\
\text { de la Educación Normal }\end{array}$ & 2005 \\
\hline Alianza por la Calidad de la Educación & 2008 \\
\hline $\begin{array}{l}\text { Reforma Educativa, Ley General de } \\
\text { Educación }\end{array}$ & $2012-2018$ \\
\hline $\begin{array}{l}\text { Ley General del Servicio Profesional } \\
\text { Docente }\end{array}$ & 2013 \\
\hline $\begin{array}{l}\text { Instituto Nacional para la Evaluación } \\
\text { de la Educación }\end{array}$ & 2013 \\
\hline $\begin{array}{l}\text { Propuesta de Reforma Educativa, Ley } \\
\text { General de Educación }\end{array}$ & 2019 \\
\hline
\end{tabular}

Tabla 2 Documentos, organismos y programas de la profesionalización docente en México

Fuente: Espinosa (2014) y complementada con información de la Secretaria de Educación Pública

Actualmente la reforma educativa del 2012-2018 menciona que se realizará un plan integral de diagnóstico, rediseño y fortalecimiento para el sistema de normales públicas, con miras a mejorar la profesionalización de los docentes y se tenga una mejora en la calidad de la educación.

El papel del docente en el nivel superior debe cumplir ciertos componentes que actualmente no están bien establecidos o claros, pero se tomaran como referentes los establecidos por la OCDE:

1. Relación entre la formación inicial y la formación continua, en este sentido se pretende que exista continuidad entre la trayectoria de formación que ha tenido el docente y la educación continua con enfoque pedagógico a través de programas de actualización que sirvan para reforzar los conocimientos de su práctica profesional que le apoyen a entender la realidad socioeducativa.
2. Uso de pedagogía centrada en el aprendizaje del estudiante, evitando que el docente se dedique solo a la transmisión de conocimientos o al uso de métodos memorísticos $\mathrm{y}$ unidireccionales, fomentando en su práctica docente la diversificación de ambientes de aprendizaje que permitan la apropiación de aprendizajes significativos

3. Práctica docente sensible a evaluación, fomentando que la práctica de los docentes sea susceptible

\section{Profesionalización docente en el nivel superior, bajo en enfoque de competencias}

La metodología de trabajo (ver figura 1) para poder revisar el impacto que han tenido las últimas reformas educativas en el nivel superior, respecto a la profesionalización docente, tanto para el caso de la Universidad Politécnica de Tlaxcala como para Escuela Normal Rural "Benito Juárez", se trabajó con datos estadísticos y se obtuvo un cálculo de muestra de docentes a los que se les aplicaría una encuesta, en la que se abordaron ítems sobre su profesionalización docente en los últimos 5 años.

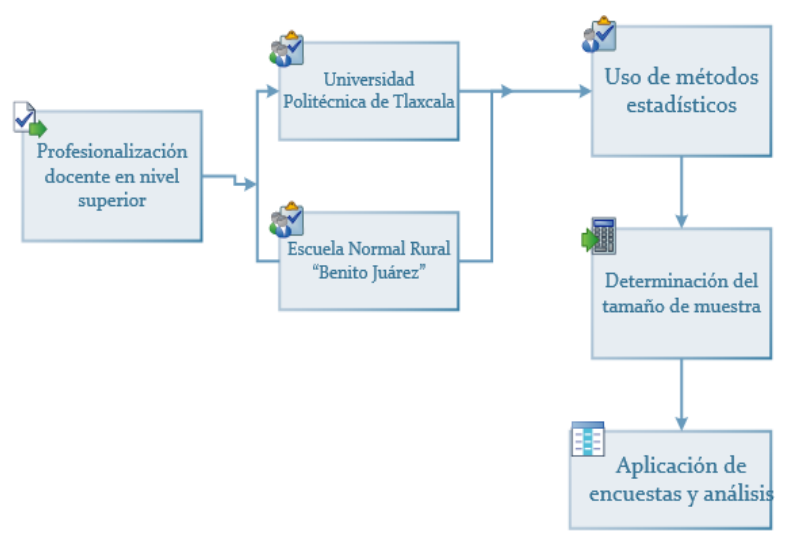

Figura 1 Metodología de trabajo

Para determinar la muestra se utilizó la fórmula 1, que es la de estadística de varianza máxima (Gómez, 1979), con una confiabilidad del $95 \%(0.05)$ y una precisión del $86 \%$.

$n=\frac{N Z^{2} p q}{(N-1) e^{2}+Z^{2} p q}$

Donde:

$\mathrm{n}=$ tamaño de muestra

$\mathrm{N}=$ tamaño de la población total

$\mathrm{Z}=$ confiabilidad con un $\alpha=1.96$

$\mathrm{p}^{*} \mathrm{q}=$ Debido a que se desconoce la probabilidad del evento se le asigna un $50 \%$ a la probabilidad de ocurrencia (p) y $50 \%$ a la probabilidad de no ocurrencia $(q)$, 
La población de docentes de los diferentes programas de estudio de la Universidad Politécnica de Tlaxcala es de 230, la medida del error es del $14 \%$ y el nivel de confianza $\mathrm{z}=1.96$ de la distribución normal, sustituyendo los valores en la fórmula 2 :

$\mathrm{n}=\frac{\left(230 * 1.96^{2} * 0.5 * 0.5\right)}{\left.\left((230-1) 0.14^{2}\right)\right)+\left(1.96^{2} * 0.5 * 0.5\right)}$

Considerando el porcentaje de docentes que aporta cada programa educativo y de acuerdo con el cálculo del tamaño de la muestra, se realizaron las encuestas que se mencionan en la Tabla 3.

\begin{tabular}{|l|r|}
\hline \multicolumn{1}{|c}{ Carrera } & $\begin{array}{c}\text { Tamaño de } \\
\text { muestra }\end{array}$ \\
\hline Ingeniería en Tecnologías de la Inf. & 6 \\
\hline Ingeniería en Biotecnología & 5 \\
\hline Ingeniería Industrial & 10 \\
\hline Ingeniería Mecatrónica & 10 \\
\hline Ingeniería Financiera & 7 \\
\hline Ingeniería Química & 6 \\
\hline
\end{tabular}

Tabla 3 Tamaño de muestra para encuesta en la UPTx. Fuente: Elaboración Propia

En el caso de la Escuela Normal Rural "Benito Juárez" la población de estudio es de 35 docentes, la medida del error es del $14 \%$ y el nivel de confianza $z=1.96$ de la distribución normal, sustituyendo los valores en la fórmula 3 :

$\mathrm{n}=\frac{\left(230 * 1.96^{2} * 0.5 * 0.5\right)}{\left.\left((230-1) 0.14^{2}\right)\right)+\left(1.96^{2} * 0.5 * 0.5\right)}$

De acuerdo con el cálculo del tamaño de la muestra, se realizaron las encuestas que se mencionan en la Tabla 4.

\begin{tabular}{|l|c|}
\hline \multicolumn{1}{|c|}{ Institución } & \multicolumn{2}{|c|}{$\begin{array}{c}\text { Tamaño de } \\
\text { muestra }\end{array}$} \\
\hline $\begin{array}{l}\text { Docentes de la Escuela Normal } \\
\text { Rural "Benito Juárez" }\end{array}$ & 6 \\
\hline
\end{tabular}

Tabla 4 Tamaño de muestra para encuesta en la E.N.R.B.J.

Fuente: Elaboración Propia

\section{Resultados}

De acuerdo con las encuestas aplicadas en ambas instituciones se observa que los resultados reales del impacto en las reformas educativas en el nivel superior no han sido satisfactorios y por ende se sigue entendiendo a la profesionalización docente como una actividad secundaria de la práctica profesional.
De esta manera se sigue destacando la formación disciplinar y el proceso de enseñanzaaprendizaje está quedando con un enfoque más tradicionalista en la transmisión de conocimientos, sin ofrecer realmente un enfoque más constructivista y que enfatice los argumentos del modelo de educación basada en competencias.

En la gráfica 1, se muestra que del total de la muestra encuestada en la UPTx, solo el $11 \%$ se encuentra realizando un diplomado enfocado en el área de pedagogía, el 54\% se encuentra o ha realizado un curso relacionado con metodologías de la enseñanza y el $23 \%$ ha realizado algún taller, finalmente el $12 \%$ no ha realizado ninguna actividad de profesionalizar su práctica docente en un periodo de cinco años.

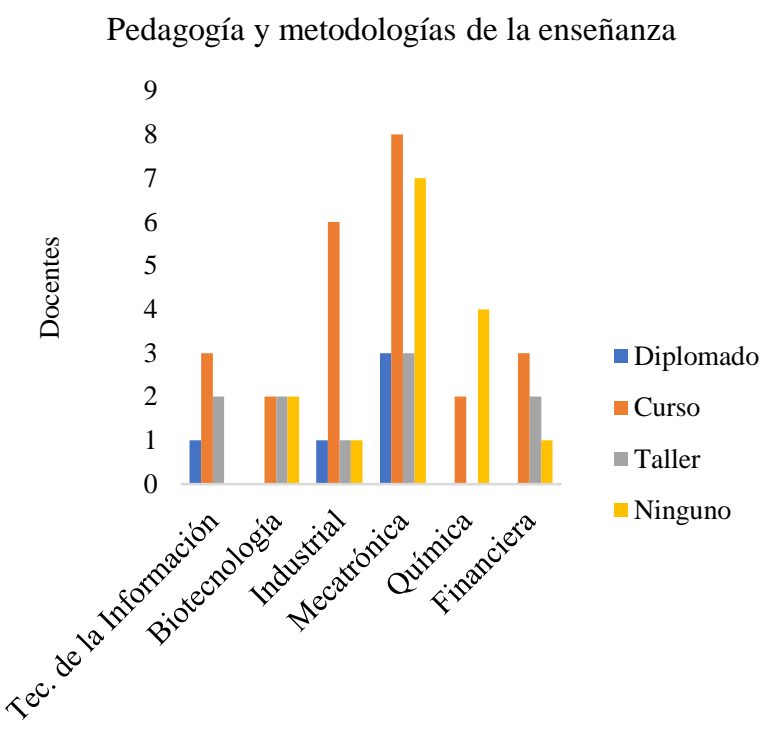

Ingenierías de la UPTx

Gráfico 1 Resultados de profesionalización docente en la UPTx

Fuente: Elaboración Propia con datos de encuesta

Con base en la gráfica anterior, se observa que en el intento de la profesionalización docente se han logrado desarrollar algunos aspectos como el dominar un saber específico o tener una preparación especializada en las áreas de ingeniería, pero hace falta que se dé le una perspectiva más integral y se convierta en un proceso en el que los docentes integren conocimientos, valores y competencias necesarias para poder ser guías y/o facilitadores de los futuros profesionistas que se están formando y que los empleadores y la sociedad están demandando. 
Aunque en el caso de la Escuela Normal Rural "Benito Juárez" las reformas educativas se han enfocado de la profesionalización docente tampoco han resultado en un cambio de concepción por parte de los docentes y se han traducido en procesos con carácter imperativo o requisitivo, que no garantizan una buena práctica profesional y tampoco se ven reflejados en la mejora de la calidad del proceso educativo.

De acuerdo con las encuestas realizadas en la Escuela Normal Rural, se muestra en la gráfica 2 que solo el $16 \%$ ha realizado algún diplomado relacionado con su profesionalización docente, el $66 \%$ ha realizado algún curso y el $16 \%$ ha asistido a algún taller en cinco años, es importante destacar entonces que esta institución formadora de docentes de nivel básico priorice sus tareas de profesionalización en función de mejorar su competitividad.

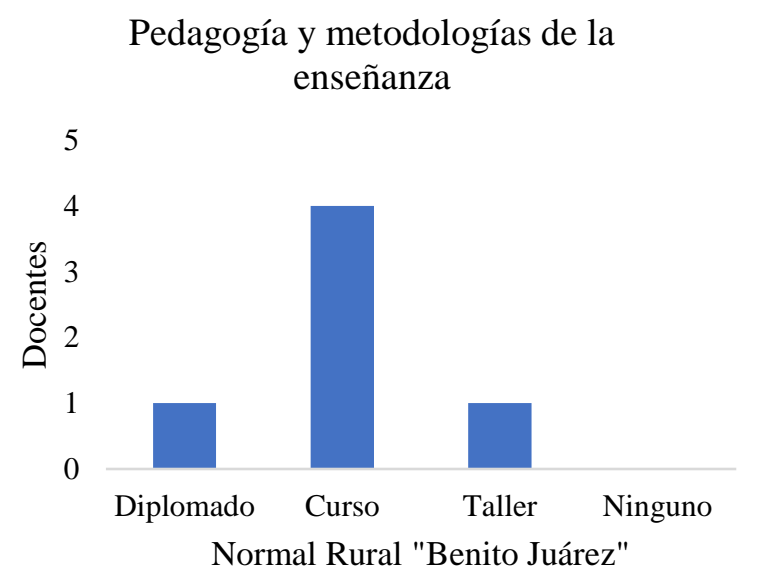

Gráfico 2 Profesionalización docente en la E.N.R.B.J Fuente: Elaboración Propia con datos de encuesta

Al realizar el comparativo y análisis de los resultados en ambas instituciones se destaca que existen puntos coincidentes y que independientemente de su enfoque de formación estudiantil, la planta docente de ambas instituciones de educación superior deben fortalecer la profesionalización de los docentes con el objetivo de mejorar los métodos de enseñanza-aprendizaje y que esto a su vez repercuta en el desarrollo de competencias y capacidades que sean de utilidad para los estudiantes, como el aprender a aprender, a través del auto-aprendizaje, o potencializar el ser de los individuos, lo cual mostraría que se están formando individuos identificados con las necesidades de su contexto y con un amplio conjunto de competencias, tal como lo remarca la educación basada en el enfoque por competencias.

\section{Agradecimientos}

A la Universidad Politécnica de Tlaxcala, por las facilidades otorgadas para la realización del trabajo.

A la Escuela Normal Rural "Benito Juárez" de Tlaxcala, por las facilidades otorgadas para la realización del trabajo.

A los docentes de ambas instituciones, que con entusiasmo participaron en las encuestas y aportaron ideas para la integración y mejora del trabajo.

\section{Conclusiones}

De acuerdo con el Instituto de administración de la educación superior de la Organización para la Cooperación y el Desarrollo Económico (OCDE) y el Centro de investigación e innovación, la mejora de calidad del sistema de educación superior en función de la situación globalizada, debe aplicar estrategias de mejora en sus tres niveles: micro (procesos de enseñanza-aprendizaje en sus lugares de desarrollo), medio (planes de estudios) y macro (políticas y estrategias institucionales).

Derivado de lo anterior, la educación en el país es el resultado de las políticas, programas y acciones que llevan a cabo los gobernantes en turno, de tal forma que aplicados a la profesionalización de los docentes se observa una discontinuidad, ya que aunque existe una evolución en este ámbito, no se han logrado alcanzar las expectativas que tales políticas y programas han perseguido.

El debate de la educación superior en México sigue haciendo énfasis en las políticas de los procesos de diseño curricular para que se pueda elevar la calidad de la educación y formar profesionistas que respondan a las necesidades del sector laboral.

Sin embargo la calidad de la educación también depende de los docentes que están al frente de la formación de los nuevos profesionistas, porque de acuerdo con los resultados obtenidos se observa que a pesar del impulso, existen contrastes entre la profesionalización de los docentes. 
Aunque bajo el enfoque del modelo educativo basado en competencias se promueve la actualización de la figura del docente, esto no ha sido del todo real, por lo que si las instituciones d educación superior enfatizara la importancia de una profesionalización adecuada, se reflejaría en una mayor efectividad en cuanto a la participación activa de los mismos en la aplicación del modelo educativo y permitiríamos que los estudiantes contribuyeran en el avance y construcción del conocimiento pedagógico y didáctico.

La revisión de las prácticas actuales de profesionalización en estas dos instituciones de educación superior demuestra que la actividad sigue siendo un suceso y no un proceso.

Una de las vertientes más prometedoras para mejorar las expectativas de profesionalización se deriva de los enfoques educativos basados en competencias, ya que en estos se destaca la figura del docente que permite demostrar el desempeño real del alumno considerando conocimientos, habilidades, destrezas, actitudes y valores involucrados en la realización de una función o actividad, lo cual derivaría de la misma acción de mejora del docente.

\section{Referencias}

Asociación Nacional de Universidades e Instituciones de Educación Superior, ANUIES (2018). Visión y Acción 2030, propuesta de la ANUIES para renovar la educación superior en México. Diseño y concertación de políticas públicas para impulsar el cambio institucional. México, D.F.

Cano Corona, A., Rojas Hernández M., Cano Corona M. (2012), Educación Basada en Competencias Profesionales para el Fortalecimiento del Sistema Educativo de Nivel Superior en el Estado de Tlaxcala, México. Memorias del VIII Congreso Internacional de Educación Superior, La Habana, Cuba.

Cano Corona M., Maldonado Bautista O., Cano Corona A., y Quiroz Macías M., (2017). Perspectivas del diseño curricular de un programa de estudios en TIC en la Educación Superior. Congreso Internacional de Educación, UAT, Tlaxcala, México.
Cañedo Ortiz, T d J; Figueroa Rubalcava, 1 E; (2013). La práctica docente en educación superior: una mirada hacia su complejidad. Sinéctica, Revista Electrónica de Educación,() 1-18. Recuperado de http://www.redalyc.org/articulo.oa?id=9982832 5003

Díaz, R. y Arancibia, V. (2002). "El enfoque de las competencias laborales: historia, definiciones y generación de un modelo de competencias para las organizaciones y las personas". Revista Psykhe, 11 (2), 207-214. Recuperado de http://www.psykhe.cl/index.php/psykhe/article/ view/433

Espinosa, J. (2014). La constitución de las prácticas de profesionalización de formación de docentes en México. Perfiles educativos, 36(143), 163-179. Recuperado, de http://www.scielo.org.mx/scielo.php?script=sci _arttext\&pid=S0185-

26982014000100010\&lng=es\&tlng=es.

Flores, P. y García, C. (2014). La Reforma Educativa en México. ¿Nuevas reglas para las IES? Revista Educativa en México, 43(172), 931. Recuperado de: http://www.scielo.org.mx/scielo.php?script=sci _arttext\&pid=S018527602014000400002\&lng $=$ es\&nrm $=$ iso

Gómez, A.R (1979), Introducción al muestreo. Tesis de maestría, Centro de estadística y Calculo, Colegio de Postgraduados, Chapingo, México.

Instituto Internacional de Investigación de Tecnología Educativa, S.C. (2010). Educación hoy: la perspectiva de la OCDE.

Pérez Ruiz, A. (2014). La profesionalización docente en el marco de la reforma educativa en México: sus implicaciones laborales. El Cotidiano, (184), 113-120. 